\title{
Towards Evolving Electronic Circuits for Autonomous Space Applications
}

\author{
Jasion D. Lohn \\ Computational Sciences Division \\ NASA Aines Research Center \\ Moffett Field, CA 94035 \\ jlohnoptolemy.arc.nasa.gov \\ Silvano P. Colombano \\ Computational Sciences Division \\ NASA Ames Research Center \\ Moffett Field, CA 94035 \\ scolombanomail arc.nasa.gov
}

\author{
Gary L. Haith \\ Recom Technologies Corp. \\ NASA Ames Research Center \\ Moffett Field, CA 94035 \\ hai thoptolemy . arc. nasa.gov \\ Dimitris Stassinopoulos \\ Computational Sciences Division \\ NASA Ames Research Center \\ Moffett Field, CA 94035 \\ stassioptolemy .arc.nasa.gov
}

\begin{abstract}
The relatively new field of Evolvable Hardware studies how simulated evolution can reconfigure, adapt, and design hardware structures in an automated manner. Space applications, especially those requiring autonomy, are potential beneficiaries of evolvable hardware. For example, robotic drilling from a mobile platform requires high-bandwidth controller circuits that are difficult to design. In this paper, we present automated design techniques based on evolutionary search that could potentially be used in such applications. First, we present a method of automatically generating analog circuit designs using evolutionary search and a circuitconstruction language. Our system allows circuit size (number of devices), circuit topology, and device values to be evolved. Using a parallel genetic algorithm, we present experimental results for five design tasks. Second, we investigate the use of coevolution in automated circuit design. We examine fitness evaluation by comparing the effectiveness of four fitness schedules. The results indicate that solution quality is highest with static and coevolving fitness schedules as compared to the other two dynamic schedules. We discuss these results and offer two possible explanations for the observed behavior: retention of useful information, and alignment of problem difficulty with circuit proficiency.
\end{abstract}

\section{INTRODUCTION}

Although the underlying concepts of using simulated evolution to manipulate hardware are decades old, it is only in recent years that research in this area has attracted significant interest $(17,6,18,19)$. The nascent field of evolvable hardware studies how simulated evolution can reconfigure, adapt, and design hardware structures in an automated manner. The field is almost ex- clusively concerned with electronic circuitry, but application areas where other types of physical structures are designed or adapted by artificial evolution certainly fall within the purview of evolvable hardware (e.g., designs of trusses, antennas).

Research on using evolution to automatically create novel circuit designs can be classified into two categories: analog and digital circuitry. In the digital circuit domain, the Field-Programmable Gate Array (FPGA) [22] has played a crucial role. The FPGA is a chip that contains a large array of logic gates (e.g., AND, OR) and a user-modifiable interconnection network to connect elements together. The distinguishing feature of the FPGA is its ability to be programmed as many times as needed. Each time the FPGA is re-programmed (the act of which is commonly called reconfiguration) for new functionality, the interconnections and gate logic change. This software-changes-hardware paradox is reconciled by the fact that the physical devices on the chip never change, only the way signals are routed through the chip changes. In practice, these chips are typically programmed to perform a desired function and are rarely re-programmed. However, in an evolvable hardware setting, theses chips are re-programmed over and over as evolution repeatedly tests new designs. The important point is that the hardware is nestled inside of the evolutionary process, allowing for rapid solution testing (a common bottleneck in many evolutionary algorithms). Some of the pioneering work in this area was done by Higuchi [5] and Thompson [21].

The other circuit design domain and the focus of this paper is analog circuitry. Analog circuits are of great importance in electronic system design since the world is fundamentally analog in nature. While the amount of digital design activity far outpaces that of analog de- 
sign. Inost digrital systems repuiro imalog moclulos for interfiuciug to the external world. Technicumes for analog circuit design antomation began appearing about two decaules ango (e.g., |20|). Efforts using techniques from evolutionary computation have appeared over the last few years. These: inclucle the luse of genetic: algorithms (GAs) $[7 \mid$ to select filter compouent sizes [8], to select filter topologices [3], and to design operational amplifiers using a sunall set of topologies [12]. Various analog filter design problems have been solved using genetic programming (e.g., [11]), and an overview of these techniques, including eight analog circuit synthesis problems, is found in $[10]$.

In space applications, automated/evolutionary design of analog circuitry could hold many benefits, especially for controller hardware. In problems where actuator outputs need to be rapidly modulated in response to sensor feedback, analog circuits have some clear advantages over digital circuits. Digital control circuits necessitate a costly bandwidth-limiting analog to digital conversion (ADC) of the sensor signal and then a reverse conversion (DAC) from the processed digital signal to the analog actuator control output. These transformations lose information and introduce latency. In many tasks the high frequency component of the sensor signal that is lost in the analog to digital conversion is crucial to usefully controlling the actuators. For example, to implement force control of a robotic manipulator, a critical portion of the strain sensor signal is lost during conversion from analog to digital. In such cases, analog circuits are ideal because they provide a very high bandwidth sensor-to-motor signal transformation and avoid any time-consuming conversion between analog and digital signals.

Although robotic controller technology has greatly improved over the last decade, certain advanced robotic applications cannot be realized due to limitations in controller processing speed, size, and power consumption. Two such applications are robotic drilling and real-time rover-astronaut interaction. Drilling from a mobile platform is difficult because the drill bit forces and position can shift rapidly causing damage to the bit - a fast force control loop could alleviate these difficulties. Roverastronaut interactions are severely limited by rover speed and autonomy, both of which would benefit greatly from fast control loops. To overcome the main limitation of using analog controllers, namely the difficulty designing analog circuits, the evolvable hardware techniques presented below could be applied to automatically generating controller circuit designs.

The remainder of the paper is as follows. First we discuss the genetic representation of analog circuits and describe the genetic algorithm that is used. Then we cover the design tasks and the experimental results from our evolutionary design program. A description of a coevolutionary method follows, including results in automated amplifier design. Lastly we discuss our conclusions relating to potential space-related applications of evolvable hatritware

\section{Circuit Representation}

In designiugr an effective circuit representation for use in evolutionary search, the following properties are among the unost chesirable. First. the representation should permit any circuit or at least a wide range of circuits to be represented. If it is known a priori that certain topologies are well suited to a specific design task, topological restrictions inherent in the representation may be beneficial since the search space will be reduced. Conversely, not having this limitation may bring to light novel designs that human designers have never envisioned. Second, the genotype conversion algorithm (the circuit constructing process) should run as fast as possible. Clearly if numerous traversals of the circuit graph structure are required in order to guarantee a valid circuit graph, the performance hit will be commensurate. For an $n$-component circuit, a reasonable upper bound would be $O(n)$. Third, the representation should be syntactically closed so that genetic operators do not create invalid circuit graphs ${ }^{1}$ from those that are valid. The circuit representation we present here was designed to have these properties.

Circuit designs are constructed by an automaton that is programmed via a set of low-level instructions. This automaton is programmed in a small "language" designed for building circuits. In its current incarnation, the language contains only component-placing instructions (e.g., control instructions are not included). This language has the desirable property that virtually all possible sequences of instructions result in a valid electrical circuit. This property is important because it greatly limits the "out-of-bounds" regions of the search space containing invalid circuit graphs. Thus, evolutionary search will spend nearly all its time generating valid circuit graphs. While this is a beneficial, non-trivial achievement, we do lose the ability to generate every possible circuit topology. This is not considered a drawback for the circuit types we investigated since a vast number of topologies and existing circuit designs could be encoded using this approach.

Each instruction places a circuit component and directs the movement of the automaton. The five basic instruction types are: $x$-move-to-new, $x$-cast-toprevious, $x$-cast-to-ground, $x$-cast-to-input, $x$-cast-tooutput, where $x$ can be replaced by $\mathrm{R}$ (resistor), C (capacitor), $\mathrm{L}$ (inductor), or transistor configuration. In a circuit design task involving only inductors and capacitors (an LC circuit), ten opcodes would be available to construct circuits (five for capacitors and five for inductors).

\footnotetext{
'Note that a graph could be a valid circuit graph, yet not make sense as an electrical circuit - for example, dissimilar voltage sources connected in parallel.
} 
The me inings of each itlstruction are summarized in Table? 1. The move-to-new instruction places one end of a component at the artive node and the other at a newly croated node (the "active" node is the current location of the automaton). The newly created node then becomes the active node. The cast-to instructions place one end of the component at the active node and the other at either the ground, input, output, or previously-created node. After executing a cast-to instruction, the automaton remains at the active node. The input and output nodes are the overall input and output nodes of the circuit as opposed to the input and output of the placed component. Illustrations of two instructions that place resistors are shown in Fig. 1.

\begin{tabular}{|l|c|c|}
\hline Instruction & Outgoing Node & Active Node \\
\hline$x$-move-to-new & new node & becomes new node \\
\hline$x$-cast-to-previous & previous node & unchanged \\
\hline$x$-cast-to-ground & ground node & unchanged \\
\hline$x$-cast-to-input & input node & unchanged \\
\hline$x$-cast-to-output & output node & unchanged \\
\hline
\end{tabular}

Table 1: Summary of opcode types used in current system. $x$ denotes the component type: resistor, capacitor, inductor, or transistor configuration.

The circuit is constructed by the automaton inside of a template circuit. The design tasks presented here use a template having one input and one output terminal as shown in Fig. 2. An ideal voltage source $v_{s}$ is connected to ground and to a source resistor $R_{s}$. The circuit's output voltage is taken across a load resistor $R_{l}$.

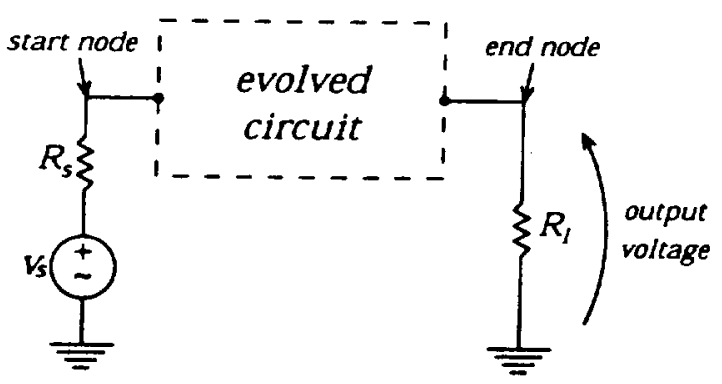

Figure 2: Template circuit: the evolved circuit is located between fixed input and output terminals. $v_{s}$ is an ideal voltage source, $R_{s}$ is the source resistance, $R_{l}$ is the load resistance.

The lists of instructions manipulated by the GA are variable-length lists so that the size of the circuit can be evolved. When the automaton reaches the last component to place in the circuit, we arbitrarily chose to have the last active node connected to the output terminal by a wire (accomplished by connection of a $1 \mu \Omega$ resistor). By doing so, we eliminate unconnected branches.

As assembly language instructions are mapped to opcodes, our circuit-placing instructions are mapped to bytecodes. Instructions are represented by up to four bytecondes. For instructions that take a component value as all argument, the first byte is the instruction, and the next. three represent the component value (resistance, capacitance, and inductance valuses). For transistors, component values are not needed. Using three bytes allows the component values to take: on one of $256^{3}$ values, a sufficiently finc-grained resolution. The raw numerical value of these bytes was then scaled into a reasonable range, depending on the type of component. Resistor values were scaled sigmoidally between 1 and $100 \mathrm{~K}$ ohms using $1 /(1+\exp (-1.4(10 x-8)))$ so that roughly $75 \%$ of the resistor values were biased to be less than $10 \mathrm{~K}$ ohms. Capacitor values were scaled between approximately $10 \mathrm{pF}$ and $200 \mu \mathrm{F}$ and inductors between roughly $0.1 \mathrm{mH}$ and $1.5 \mathrm{H}$.

Transistors are current amplifying and switching devices that have three terminals. ${ }^{2}$ In this paper we use bipolar junction transistors as shown in Fig. 3. Using devices with three terminals makes it harder to design a circuit representation that achieves the properties that we desire. If a circuit constructing automaton were to connect one terminal of a transistor at a node, then two active nodes would result each requiring its own automaton. This could happen repeatedly resulting in an exponential growth of automata constructing the circuit in parallel. Two problems are obvious: how will the multiple constructing "threads" interconnect, and how will the dangling nodes that will likely appear at the end of the circuit constructing process be handled? To allow interconnections between constructing threads, one can introduce a spatial dimension and let the automata form interconnections as they criss-cross each other's path. To handle the dangling node problem, one can determinstically tie dangling nodes to each other, to internal nodes, or to the output node for example. Another solution is to simply prune those nodes. Although we have considered these and other solutions, a simpler way of handling transistors is also a viable alternative: treating them as having only two terminals.

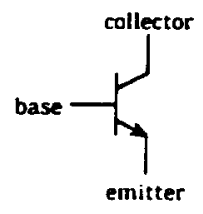

(a)

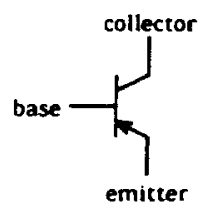

(b)
Figure 3: Bipolar junction transistor symbols: (a) npn; (b) pnp.

To work with transistors as devices with two terminals, we have the third terminal hardwired (fixed) to one of the following pre-existing circuit nodes: ground, power supply (positive or negative), input, output, the

\footnotetext{
${ }^{2}$ Four if the subatrate terminal is included, but we connect the subetrate to ground and hence ignore it.
} 


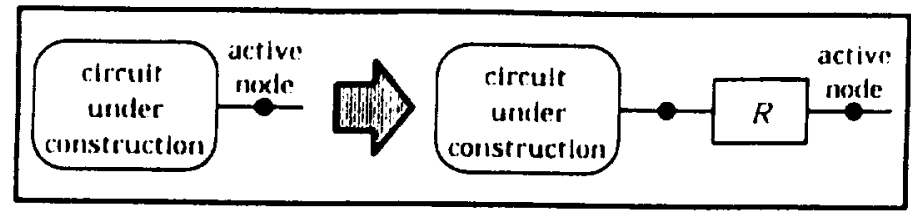

result of move-to-new instruction

(a)

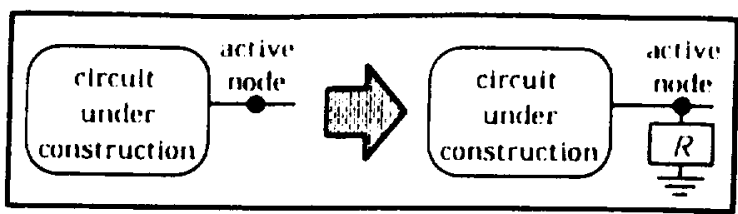

result of cast-to-ground instruction

(b)

Figure 1: Effect of placing a resistor with (a) move-to-new, and (b) cast-to-ground instructions.

previously placed node, or even to itself. Such a scheme allows a wide variety of configurations. To understand these configurations we label the terminals in a generic way: incoming, outgoing, and fixed (see Fig. 4). The incoming terminal is the terminal that the circuit constructor will connect to the active node. The outgoing terminal will become the new active node (for move-to instructions) or it will be cast to a pre-existing circuit node. The fixed terminal is hardwired as its name implies. (a)

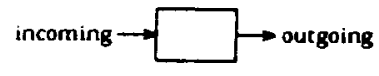

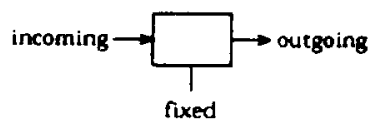

(b)
Figure 4: Labeling of terminals: (a) devices with two terminals have incoming and outgoing terminals; (b) devices with three terminals are treated as two-terminal devices by having a fixed connection at the third terminal.

To give a sense of the types of transistor configurations possible, the chart in Fig. 5 illustrates 52 configurations for an npn transistor whose base terminal is designated incoming. Each entry shows the connections that the automaton makes when executing the instruction listed in the first column. The last two columns show self-connections, some of which are frequently used by circuit designers. Similar charts can be produced for npn transistors having the collector and emitter serve as the incoming terminal, as well as the three analogous charts for pnp transistors. There are configuration redundancies so that each chart will not have exactly 52 configurations. In addition we exclude emitter-collector self-connections since this shorts out the transistor.

This approach to representing circuits embodies the desirable properties outlined above. The encoding has syntactic closure since any combination of instructions produces a valid circuit graph, and since every instruction contained in the genome results in a circuit component, there are no non-coding genome segments. The circuit construction process is $O(n)$ since it does not require any repair (e.g., removal of unconnected nodes) operations. Lastly, this approach can generate a wide range of circuit graph topologies. The topological restriction is as follows: circuit branches off of the main constructing thread camnot, in general, contain more than one node (there are some exceptions to this). The constructing thread is the sequence of components that are created by the move-to-new instructions. The constructing thread itself can be of varying lengths and can contain both series and parallel configurations. In spite of these limitations, our system allows the creation of circuits with a large variety of topologies, including numerous topologies seen in hand-designed circuits.

\section{DESIGN TASKS}

The design tasks considered in this paper are analog circuts for filtering and amplification applications.

A low-pass filter is a circuit that allows low frequencies to pass through it, but stops high frequencies from doing so. In other words, it is frequency selective in that it "filters out" frequencies above a specified frequency. The unshaded area in Fig. 6 depicts the region of operation for low-pass filters. Below the frequency $f_{p}$ the input signal is passed to the output, potentially reduced (attenuated) by $K_{p}$ decibels (dB). This region is known as the passband. Above the frequency $f_{s}$, in the region is called the stopband, the input signal is markedly decreased by $K$, decibels. Between the passband and stopband the frequency response curve transitions from low to high attenuation. The parameter located in this region, $f_{c}$, is known as the cutoff frequency.

One of the design tasks concerned designing a circuit within the class of "Butterworth" filters. Butterworth filters are very common and circuits that implement them are readily found in filter design tables [9]. The attenuation (negative gain) of Butterworth filters is of the form $\sqrt{1 /\left(1+\left(f / f_{c}\right)^{2 N}\right)}$ where $f$ is the input frequency and $N$ is the order of the filter. The higher order a filter has, the sharper the "knee" of its gain curve, and the more complex the circuit. A plot of the attenuation for a third-order Butterworth filter is shown in Fig. 7.

The amplifer design task chosen was the inverting operational amplifier. Such a circuit has found wide application and is considered one of the workhorses of analog circuit design. Figure 8 shows the symbol and connections for an ideal inverting amplifier. This circuit generates an output voltage $\left(v_{o}\right)$ that consists of the input 


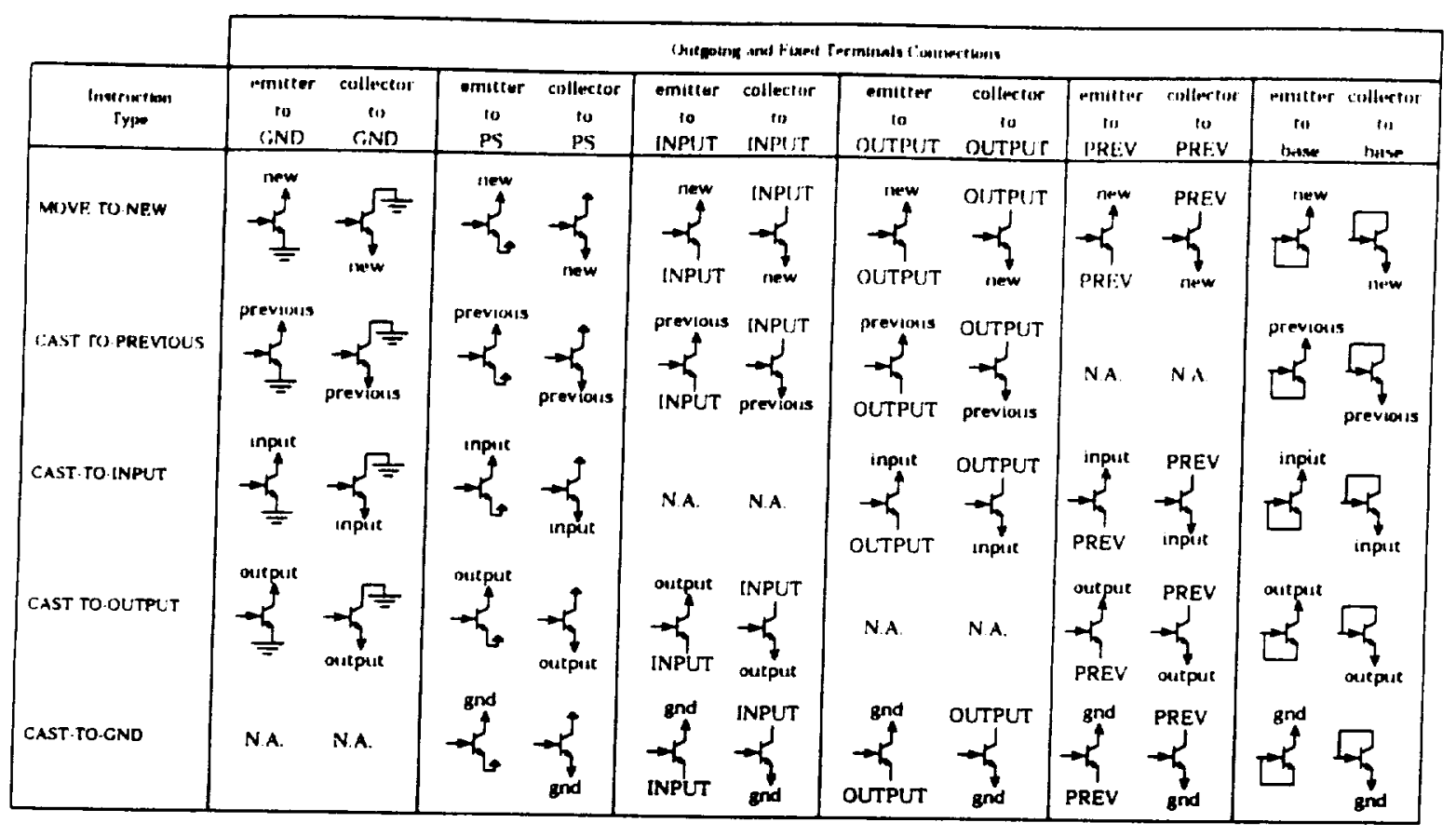

Figure 5: Transistor configurations showing the outgoing and fixed connections when the incoming terminal is the transistor's base terminal. Terminals labeled in upper case letters denote the fixed terminal connection. In the last two columns the fixed connection is a self-connection. "PS" denotes the power supply (only the positive version is shown), and "N.A." denotes "not applicable." Only npn transistors are shown although analogous configurations are present for pnp transistors.

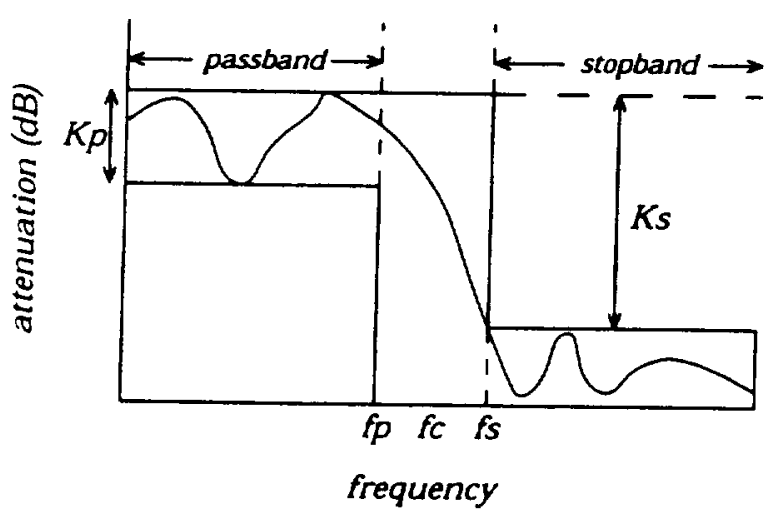

Figure 6: Low-pass filter terminology and specifications. The shaded regions represent out-of-specification areas. An example frequency response curve that meets specifications is shown.

voltage $\left(v_{i}\right)$ multiplied by a gain factor, $A$. Voltage gain is thus equivalent to $v_{o} / v_{i}$. It is common to express gain values in decibels $(\mathrm{dB})$ using $20 \log _{10}(A)$. Amplifiers may be either inverting or non-inverting, where an inverted output signal has a $180^{\circ}$ phase shift compared to the input. The dc gain of the amplifier refers to the gain when only constant voltage/current sources are applied. The linearity of the gain is the degree to which the gain remains constant across input voltages: ideally the voltage transfer characteristic ( $v_{0}$ vs. $v_{i}$ ) should be linear. The dc component that shifts the entire signal up or down is

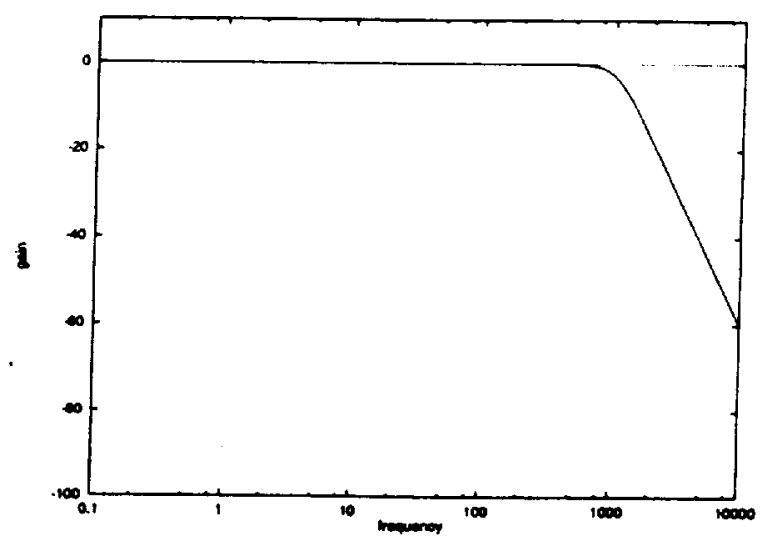

Figure 7: Gain on a logarithmic amplitude scale for a third-order Butterworth filter.

called the dc bias of the circuit. Power dissipation is the amount of power used by the circuit and is indicative of the amounts of current flowing in the circuit. For simple amplifiers, there are publications available that catalog many designs. Since there are numerous parameters in amplifier design (e.g., input/output impedance, power dissipation, distortion, common-mode rejection, power supply rejection), the design task can become quite chatlenging and typically requires an experienced designer. For the amplifier design experiments below, we take into account four objectives: dc gain, linearity of gain, de bias, and power dissipation. 


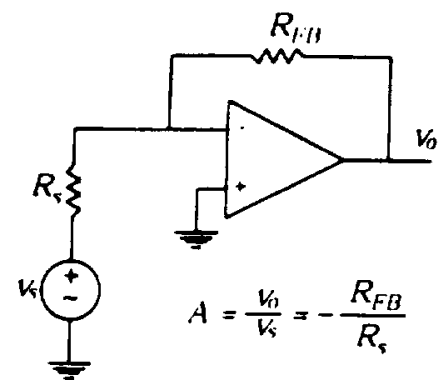

Figure 8: Ideal inverting amplifier showing how gain is set by the ratio of the feedback to source resistor.

\section{Experimental Results}

\section{Filter Design Tasks}

Three filter design experiments were performed. In each experiment, 10 runs were performed and we present the circuit having the bighest fitness value across all runs. The experiments increased in difficulty so that filter 3 represents a challenging design task, while filter 1 is least challenging. Table 2 lists the target specifications for each of the experiments.

\begin{tabular}{|c|c|c|c|c|}
\hline Filter No. & $f_{P}(\mathrm{~Hz})$ & $f_{s}(\mathrm{~Hz})$ & $K_{p}(\mathrm{~dB})$ & $K_{s}(\mathrm{~dB})$ \\
\hline \hline 1 & 100 & 4000 & 1.29 & 27.12 \\
\hline 2 & 925 & 3200 & 3.01 & 22.00 \\
\hline 3 & 1000 & 2000 & 0.01 & 63.50 \\
\hline
\end{tabular}

Table 2: Target specifications for filter design tasks.

The GA parameters remained the same within a given experiment, but varied in the number of evaluations (circuit simulations): filter 1 runs had 30,000 evaluations, filter 2 had 3.6 million, and filter 3 had 1 million. These values were arrived at by experimentation and constrained by practical issues such as the availability of workstations.

For the filter experiments, fitness was calculated to promote the regression of the evolved circuit's frequency response toward that of the target. Error values were computed as the absolute value of the difference of the individual's output and the target output. These error values were summed across evaluation points to arrive at a fitness value.

Electronic Stethoscope Circuit - The first filter design task was set up to generate a filter suitable for use in an electronic stethoscope. In this application, it is desired to filter out the extraneous high-frequency sounds picked up by a microphone which make it difficult to listen to (low-frequency) bodily sounds (e.g., a heart beating). As such, the frequency response specifications do not need to be extremely accurate since the human ear cannot discern frequencies that are close together. The target frequency response diatia was taken from in anctual electronic stethoscope, which wis built with a cutolf frequency of $796 \mathrm{~Hz}$ corresponding to an output voltage of approximately 1 volt. This circuit is relatively easy to design and so we chose it as our first design task. The cc-bot instruction set consisted of ten instructions, five for resistors and five for capacitors, which allowed for the construction of an RC low-pisss filter. The evolved circuit is shown in Fig. 9(a) and its frequency response, which matches almost exactly the target is shown in Fig. 9(b). It was found in generation 3 of a 10-generation run that had a population size of 3000 , an indication that this design task was relatively easy. The circuit exhibits the standard design for simple low-pass filters: a resistor $\left(R_{2}\right)$ in series with the source to form a voltage divider at low frequencies ( $C_{1}$ open), and a capacitor $\left(C_{1}\right)$ across the output to short it at high frequencies.

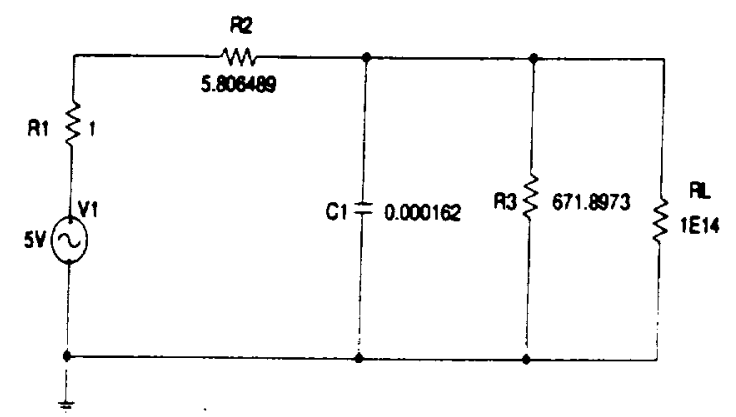

(a)

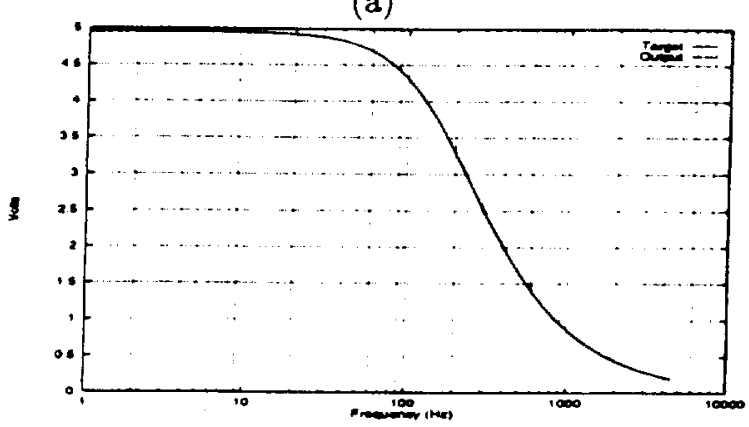

(b)

Figure 9: (a) Evolved low-pass filter for use in an electronic stethoscope (units are ohms and farads); (b) Nearly identical frequency response curves for evolved and actual electronic stethoscope circuit. The frequency axis is scaled logarithmically.

Butterworth Low-pass Filter - The second low-pass filter design task had specifications that were more difficult to achieve than the first filter: both the passband and the stopband were longer, thus requiring the transition to be sharper. We chose a circuit that can be built uring a 3rd-order Butterworth filter and having a frequency response of the form seen in Fig. 7. The specifications are listed under filter number two in Table II.

Such a filter design can be derived using a ladder topol- 
ogy (ont:aning two capacitors and one: inductor and component vinlues found in publistued tables. Because we wanted to design an LC low-pass filter, the cc-bot instruction set consisted of only capacitor and inductor instructions. The evolved circuit that meets these specifications is shown in Fig. 10 and its frequency response is shown in Fig. 11. It was found in generation 22 of a run that had a population size of 18,000 .

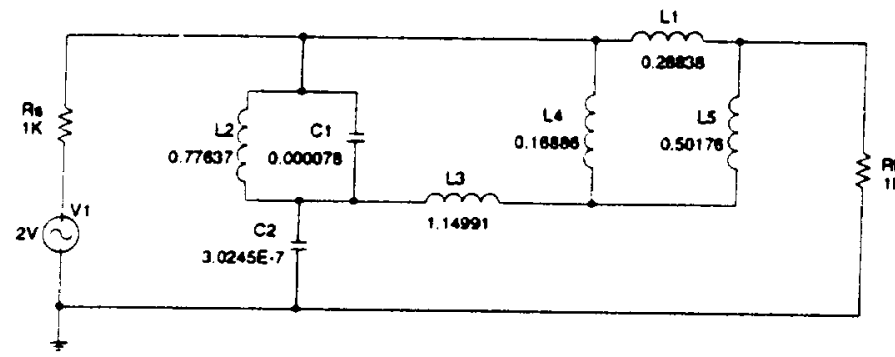

Figure 10: Evolved 3rd-order Butterworth low-pass filter (units are ohms, farads, and henries).

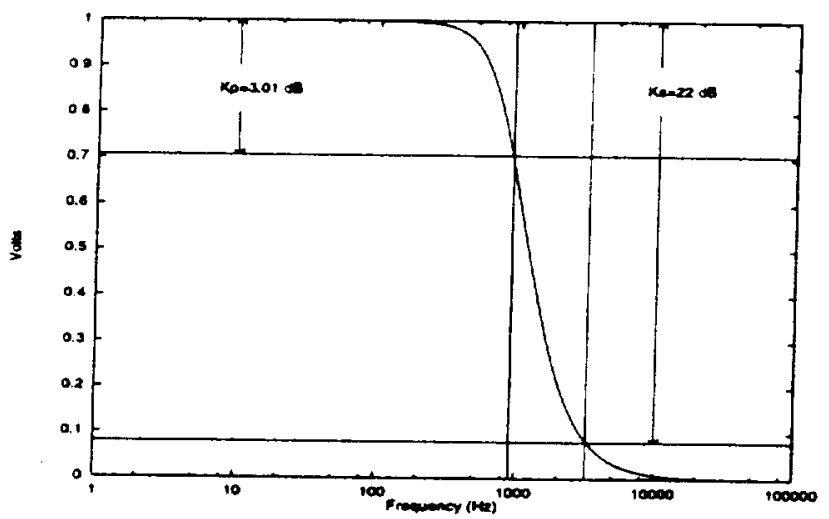

Figure 11: Frequency response curve for evolved 3rdorder Butterworth low-pass filter. Attenuation specifcations are also shown. The frequency axis is scaled logarithmically.

Third Low-pass Filter - The third low-pass filter design task had specifications that were the most stringent: in addition to the passband and stopband being increased, the attenuation parameters were tightened (see Table II). These specifications are similar to the ffth-order elliptic filter described in [10]. In that work, the evolved LC circuit satisfies $K_{p}=0.3 \mathrm{~dB}$ and $K_{\bullet}=60 \mathrm{~dB}$. Another evolved low-pass filter circuit [23] had the same stopband and passband frequencies, but less demanding attenuation specifications $\left(K_{p}=1.6 \mathrm{~dB}\right.$ and $\left.K_{0}=24.8 \mathrm{~dB}\right)$. The evolved circuit is shown in Fig. 12 and its frequency response is seen in Fig. 13. Micro-ohm resistors were added as a convergence aid for the circuit simulator, and can be ignored for analytical purposes. This circuit was found in gemeration 997 of a run that had a population size of 1000. The ce-bot instruction se(uence and SPICE netlist for this circuit are included for reference in the Appendix.

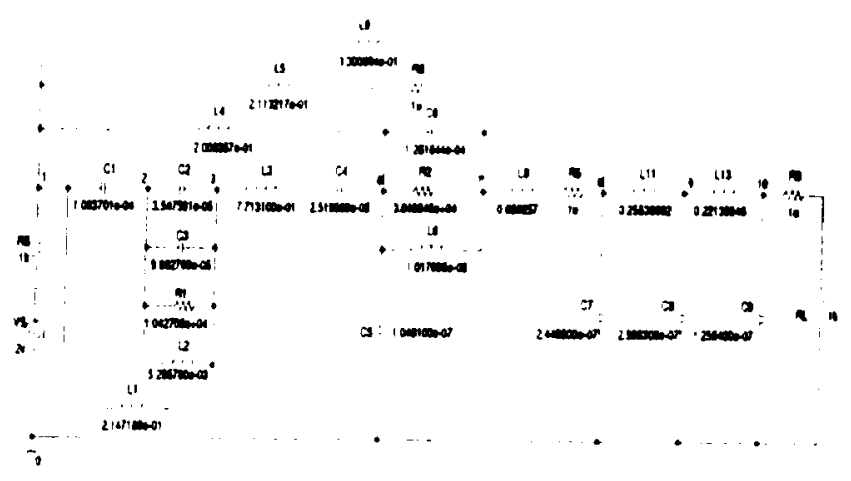

Figure 12: Evolved circuit satisfying target specifications for filter number three.

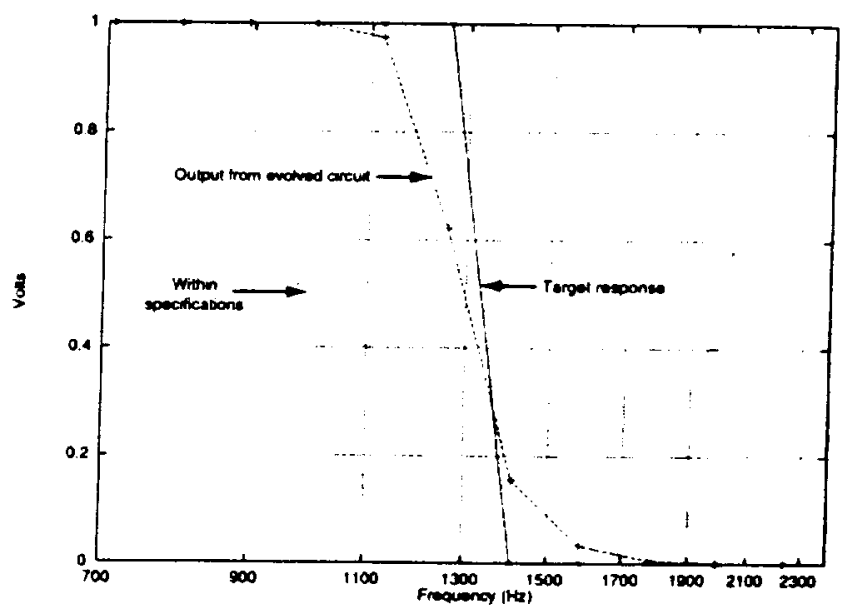

Figure 13: Frequency response for filter number three.

\section{Amplifier Design Tasks}

Two amplifier design experiments were performed. In each experiment, 10 runs were performed and we present the highest performance circuits found across all runs. The goal was to design an inverting amplifier capable of a dc voltage gain up to a maximum of either $100 \mathrm{~dB}$ or $120 \mathrm{~dB}$, while minimizing $\mathrm{dc}$ bias and maximizing linearity over the dc gain. Population size was set to 1200 individuals, and each run proceeded for 5000 generations, giving a total of 6 million circuit evaluations per run. The difference between the two sets of experiments is that in the first set, the maximum gain was set to be $120 \mathrm{~dB}$, and in the second set, $100 \mathrm{~dB}$ was the maximum gain. The maximum gain possible is set by using feedback resistors (labeled $R_{F B}$ ). For an ideal inverting amplifier (as shown in Fig. 8), the magnitude of the gain of the amplifier is simply $R_{F B} / R_{S}$, where $R_{S}$ 
is the source resistor. Fitness wibs calculated in a manner similar to the: work on amplifiers in [10]. An error value is computed as the sum of the dc gain penalty (the target gain minus the observed gain), the de bias (zero de: bias is idcal), and the (legrey to which the do: gain is limear.

7.5 (IB Inverting Amplifier - In the first set of experinents the maximum voltage gain was set at $120 \mathrm{~dB}$ $\left(10^{6}\right)$. The amplifier having the best performance had a dc gain of $74.53 \mathrm{~dB}$ (5324.40). Figure 14 shows the schematic for this circuit. It was found in generation 4866, and had a dc bias of 3.64 volts and a power dissipation of 0.82 watts.

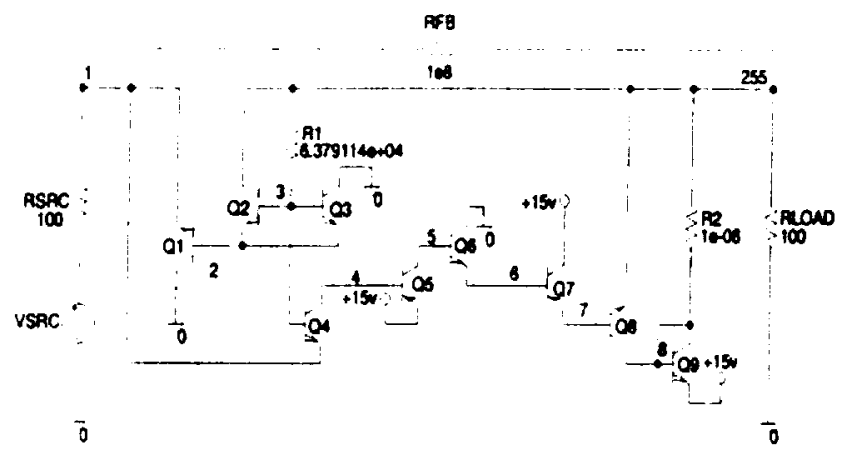

Figure 14: Circuit schematic of evolved $75 \mathrm{~dB}$ amplifier.

The dc behavior is best understood by examining the major current pathways in the circuit. The current through the load is the key quantity since it is converted to a voltage by the load resistor and hence forms the circuit's output. Nearly all of the dc current flowing through the load resistor originates from the power supply connected to transistor Q7's collector. Q7 is biased in such a way as to supply Q8's base with approximately $36.4 \mathrm{~mA}$ of current. This current is divided so that $18.1 \mathrm{~mA}$ flows out of Q8's emitter and $18.3 \mathrm{~mA}$ out of Q8's collector. Resistor R2 is a tiny resistance that was positioned in order to connect transistor Q9 to the output (the last component is forced to connect to the output terminal). Thus R2 can be ignored, and transistor Q9's $18.4 \mathrm{~mA}$ current flows into the output node. Currents are summed at node 255 to give the load current of $36.4 \mathrm{~mA}$ which flows through the load resistance to give 3.64 volts output. Because there is a negligible amount of current flowing through transistors Q1 through Q4, the utility of these transistors is unclear. Components that are essentially non-functional, are quite commonly seen in evolutionary design applications. Figure 15(a) shows the time domain response. Amplification of a $1 \mathrm{kHz}$ sine wave having a 1 microvolt amplitude can clearly be seen. Figure 15(b) shows the frequency response. The ac gain remains flat at $74.36 \mathrm{~dB}$ until it loses $3 \mathrm{~dB}$ at $7.59 \mathrm{kHz}$ (its $3 \mathrm{~dB}$ bandwidth). Figure 16 shows the dc transfer characteristic. The dc bias of 3.64 volts can be seen at the voltage input of zero volts.

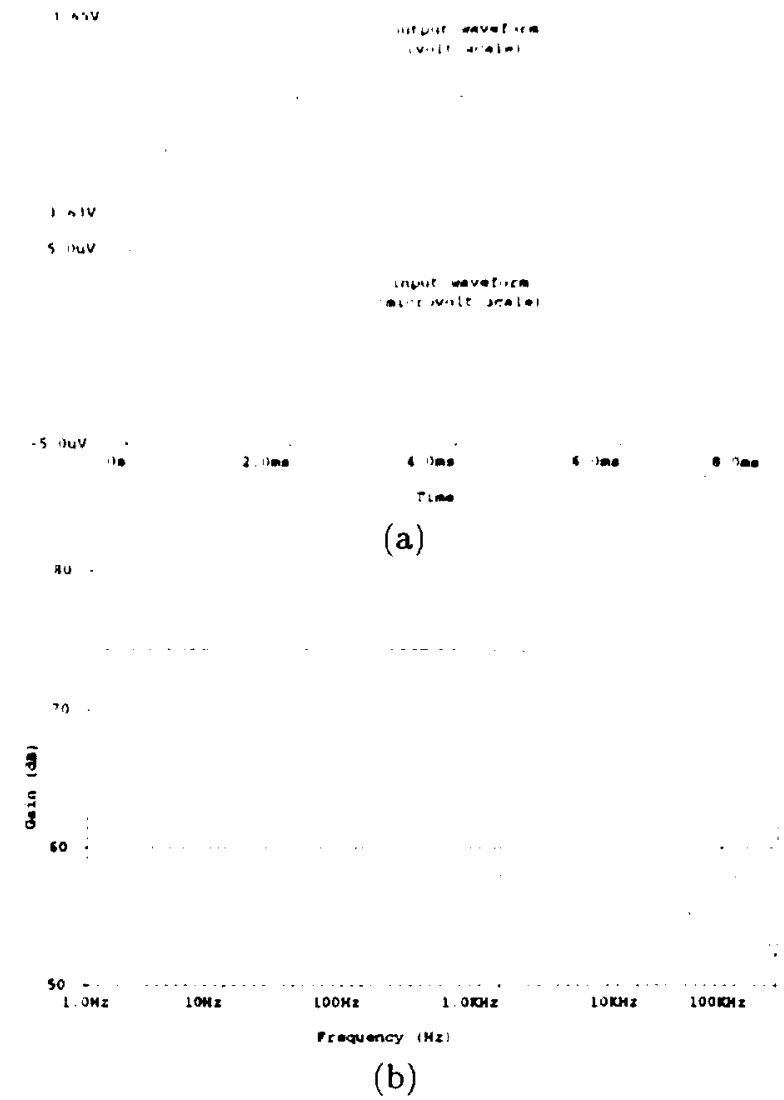

Figure 15: Small signal behavior of $75 \mathrm{~dB}$ evolved amplifier: (a) time domain input waveform is $1 \mathrm{kHz}$ (bottom) which is inverted and amplified (top); (b) frequency response showing $3 \mathrm{~dB}$ bandwidth of $7.59 \mathrm{kHz}$.

$85 d B$ Amplifier - In the second set of amplifier experiments the maximum voltage gain was set at $100 \mathrm{~dB}$ $\left(10^{5}\right)$. The amplifier having the best performance had a dc gain of $85.41 \mathrm{~dB}(18,642.33)$. Figure 17 shows the schematic for this circuit. It was found in generation 3635 , and had a dc bias of 5.44 volts and a power dissipation of 8.17 watts. The dc current delivered to the load is mostly supplied by the 15 volt battery attached to the collector of transistor Q7. Transistor Q7 is conducting with the sum of its base and collector currents flowing out of its emitter. Q7's base current of $13 \mathrm{~mA}$ is supplied by transistor Q6. As in the previous amplifier, the utility of transistors Q1 through Q3 is unclear.

Input signal inversion and amplification are seen in Figure 18(a) which shows the time domain response to an ac input of 1 microvolt at $1 \mathrm{kHz}$. The circuit has a flatband gain of $85.46 \mathrm{~dB}$ and $\mathrm{a} 3 \mathrm{~dB}$ bandwidth of $282.8 \mathrm{kHz}$ (Figure 18(b)). The $3 \mathrm{~dB}$ bandwidth is significantly better than the previous amplifier. Figure 19 shows the dc transfer characteristic. The dc bias of 5.44 volts can be seen at the voltage input of zero volts. The slope, the magnitude of which is the gain, is negative since the amplifier is inverting the signal. 


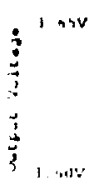

1558.1048 $.5 w^{\prime \prime}$ Inpue vol toge suv

Figure 16: DC transfer characteristic of $75 \mathrm{~dB}$ amplifier.

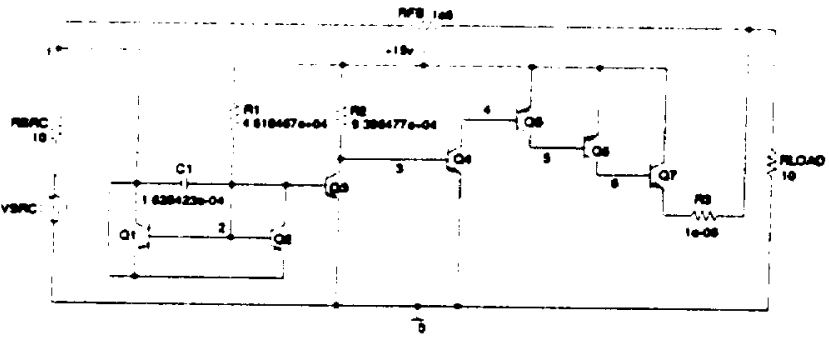

Figure 17: Circuit schematic of evolved $85 \mathrm{~dB}$ amplifier.

\section{Coevolution and Other Fit- NESS SCHEDULES}

Using the same amplifier design task described above, we now turn our attention to comparing four methods of evolutinary search. A fitness schedule is contruct that modifies how a circuit's fitness is computed during the GA run. For example, one could use a fitness function $f_{1}$ during generation $1, f_{2}$ during generation 2 , etc. Four fitness schedules, one of which embodies coevolutionary concepts, are discussed below.

The first, called static for short, refers to a single fitness function that is used to evaluate every individual in every generation of the run. The second was a fixed fitness schedule, meaning that the fitness function was modified in a pre-determined manner every $k$ generations, for some constant $k$. Thus the same fitness function evaluates groups of $k M$ individuals, where $M$ is the population size. The third fitness schedule we call adaptive because it can change the fitness function dynamically based on the performance of the population. The fourth fitness schedule is coevolutionary search whereby a second of population consisting of problem difficulties (target vectors) evolve based on the performance of the circuits in the main population.

\section{Static Fitness Schedule}

The static fitness schedule is simply the standard evaluation technique in genetic algorithms (7): a single fitness
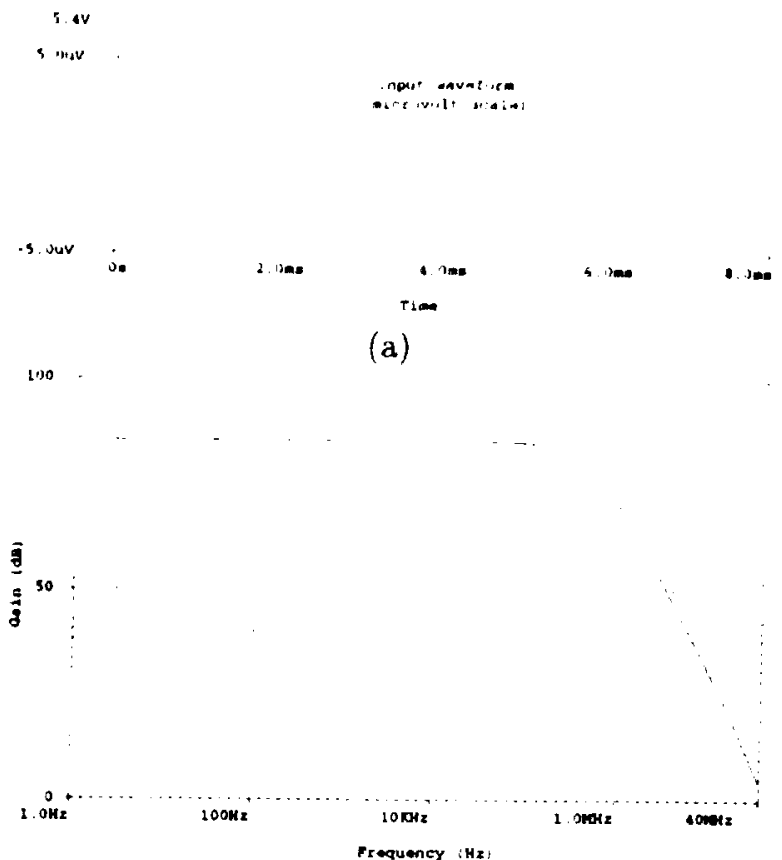

(b)

Figure 18: Small signal behavior of $85 \mathrm{~dB}$ evolved amplifier: (a) time domain input waveform is $1 \mathrm{kHz}$ (bottom) which is inverted and amplified (top); (b) frequency response showing a flatband gain of $85.46 \mathrm{~dB}$.

function is used to evaluate all individuals throughout the run. The fitness function used is similar to those described in $[10,13]$. Briefly, it is a sum of normalized error values, where the errors are the shortfalls from the desired objectives: dc gain, dc bias, power dissipation, and the linearity of the dc gain. The gain is the slope of the dc transfer characteristic (i.e., the output voltages when the input voltage is swept across five input voltages). The slope, $m$, is calculated by using the endpoints of the transfer characteristic. The linearity of the gain is computed as $\left|m-m_{l}\right|+\left|m-m_{r}\right|$, where $m_{l}$ is the slope of the line segment formed by the two leftmost output voltages and $m_{r}$ is analogous for the two rightmost output voltages. The de bias is simply $v_{0}$ when $v_{i}=0$ volts, and power dissipation is the amount of power consumed during circuit operation. The gain objective was $60.0 \mathrm{~dB}$, the bias and power dissipation objectives were 1.0 volt and 1.0 watt, respectively, and the linearity objective was 10.0. These values were chosen based on our previous work [14]: they represent a moderately difficult design task that we knew to be solvable. 
Figure 19: DC Transfer characteristic of $85 \mathrm{~dB}$ amplifier

\section{Fixed Fitness Schedule}

The fixed fitness schedule is a pre-determined schedule of fitness function modifications. As used in the experiments below, the difficulty-level of the fitness function is increased every 50 generations. With a total of 5000 generations, this allowed for a total of 100 udifficulty steps." Each of the fitness functions used over the course of the run are of the same form as the fitness function used in the static schedule above. Writing our gain, bias, power, and linearity objectives as a target vector, $\langle G, B, P, L\rangle$, we specified that the difficulty level begins at $\langle 1.0,10.0,10.0,1000.0\rangle$ (easiest) and ends at $\langle 60.0,1.0,1.0,10.0\rangle$ (most difficult). The increases in difficulty are then evenly divided over the 100 steps, per objective. This is admittedly an arbitrary schedule, but that is an inherent property of a fixed schedule - it is subject to the biases of the implementor. Such biases can be advantageous if knowledge of the fitness landscape is known a priori, and potentially disadvantageous otherwise.

\section{Adaptive Fitness Schedule}

The adaptive fitness schedule is identical to the fixed schedule described above except in the following regard: difficulty is incremented "on-demand," whenever the current difficulty is solved by at least one circuit in the population. As in the fixed schedule case, 100 difficulty steps are provided for. If a circuit solves the 100th fitness function before $\mathbf{5 0 0 0}$ generations, it has successfully found a compliant circuit, and the run halts. On the other hand, if 5000 generations elapse and a compliant circuit is not found, the run halts at whatever difficulty level it has reached.

\section{Coevolving Fitness Schedule}

The main difference between the coevolving fitness schedule and the other dynamic schedules is the introduction of a second population consisting of target vectors ( $t v)$. The first population of circuits remains the satme as in the ether fituress scherlules. The t:atrget vector population consists of individuals that specify problem difficulty. As described above, target vestors are denotexl $\langle G, B, P, L\rangle$, representing gain, bias, power dissipation, and gain linearity, respectively. The individual targets are threshold values - a target is "solved" if a circuit's performance equals or surpasses (either above or below, as appropriate) the threshold specified. For example, $\langle 63.0,0.6,0.8,9.5\rangle$ solves $\langle 60.0,1.0,1.0,10.0\rangle$, but $\langle 58.0,0.6,1.2,18.0\rangle$ does not. As with the other fitness schedules, the ideal target vector used was $\langle 60.0,1.0,1.0,10.0\rangle$. The gain target is satisfied if a circuit's gain was 60.0 decibels or greater. The three remaining targets were satisfied if the circuit's performance is less than or equal to the target values.

Target vectors are represented as a list of floating point values that are mutated individually by randomly adding or subtracting a small amount (5\% of the largest legal value). Single point crossover was used, and crossover points were chosen between the values.

Fitness of individual circuits in the main population was computed as follows. Circuit $i$ "plays" each target vector in the second population and a score, $s_{i}$, is computed:

$$
s_{\imath}=\sum_{j \in \widehat{t v_{1}}} \frac{1}{\begin{array}{c}
\text { total \# circuits } \\
\text { that solve tv } j_{j}
\end{array}}
$$

where $\widehat{t v}_{i}$ is the set of target vector indexes such that circuit $i$ solves $\mathrm{tv}_{j}$. Note that the denominator in the above fraction is guaranteed to be greater than or equal to one due to the restriction on $j$. Then $s_{i}$ is normalized linearly between its upper and lower bounds such that 0.0 is the best score and 1.0 the worst:

$$
F\left(\text { circuit }_{i}\right)=1.0-s_{i} / M_{2}
$$

where $M_{2}$ is the size of the target vector population. The effect of $s$ is to reward circuits that solve the more difficult target vectors. A target vector has the greatest difficulty level when exactly one circuit can solve it. If many circuits can solve a particular target vector, the fitness contribution in $s$ is shared among the circuits [16].

Fitness of an individual target vector is computed as follows. Let $x_{j}$ denote the number of circuits that solve $\mathrm{tv}_{j}$, and $M_{1}$ be the circuit population size. The fitness is essentially $x_{j}$, scaled and normalized, with a tractability constraint:

$$
F\left(\mathrm{tv}_{j}\right)=\left\{\begin{array}{cc}
1.0 & x_{j}=0 \\
\frac{1}{\left(M_{1}-1\right)}\left(x_{j}-1.0\right) & x_{j} \geq 1
\end{array}\right.
$$

The tractability constraint gives a target vector a score of 1.0 (the "worst" score) when no circuits can solve it. This puts pressure on the target vector population to pose difficult, yet solvable problems to the circuit population. 


\begin{tabular}{|c|c|c|c|c|}
\hline 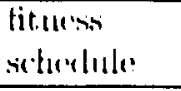 & $\begin{array}{l}\text { gitin } \\
\text { [IB }\end{array}$ & $\begin{array}{c}\text { biats } \\
\text { [volts } \mid\end{array}$ & $\begin{array}{l}\text { powwer } \\
\text { [watts! }\end{array}$ & $\begin{array}{l}\text { linearity } \\
\text { [unitless) }\end{array}$ \\
\hline st.atici: & 44.47 & 0.355 & (0.69) & 49.28 \\
\hline tixted & 47.59 & 0.64 & 1.21 & 96.633 \\
\hline inlitptive: & 54.1 .3 & 1.23 & 1.96 & 340.74 \\
\hline coevolving & $46.7 !$ & 0.15 & 0.41 & 189.75 \\
\hline
\end{tabular}

Table 3: Mean values from the performance of the best circuits found under 25 runs of each fitness schedule. The ideal target vector was $\langle 60.0,1.0,1.0,10.0\rangle$.

\section{Experimental Setup}

Using the four fitness schedules described above, 25 runs using each schedule were made resulting in a total of 100 runs. The same pseudo-random number generator seed was used across each set of four distinct fitness schedules so that the generation zero individuals would be identical. Common to each run were the following parameter settings: population size was 600 , crossover rate was $80 \%$, mutation rate was $5 \%$. For the coevolution runs, the target vector population used the following parameters: population size was 600 , crossover rate was $80 \%$, mutation rate was $50 \%$. Because crossover points were chosen between target vector values, this mutation rate was set high to encourage new values to appear in the population, not just those produced in generation 0 .

Evolution of amplifier designs was accomplished using the system described in [13]. Briefly, circuits are represented as lists of circuit-construction instructions that program an automaton to design a circuit. Resistors, capacitors, and bipolar junction transistors were the allowed components. The method of incorporating transistors is described in [14]. Circuits were required to contain at least 10 components up to a maximum of 150 .

\section{EXPERIMENTAL Results}

To assess the quality of each fitness schedule, we examined the highest fitness circuits from each run. The performance of these circuits is quantified in corresponding output vectors which, like target vectors, specify gain, bias, power dissipation, and linearity values. Table 3 gives the mean values of individual objectives across output vectors for each fitness schedule. The data suggest that static and coevolving fitness schedules performed better than fixed and adaptive schedules. Another way of measuring the quality of the fitness schedules is to look at the number of objectives solved in each run (assuming each of the four objectives is of equal importance). Table 4 shows the mean and standard deviation for the number of objectives solved for each schedule.

Here the relationship among the schedules is clearer: static and coevolving fitness schedules performed nearly the same and did better than the performance of the

\begin{tabular}{|c|c|c|}
\hline $\begin{array}{l}\text { fituness } \\
\text { schoululo? }\end{array}$ & miriblu & stal. dev. \\
\hline stantic: & $2.12^{*}$ & 0.677 \\
\hline fixerl & 1.48 & 1.12 \\
\hline alaptive? & 1. 16 & 1.18 \\
\hline coevolving & $2.08^{*}$ & 0.49 \\
\hline
\end{tabular}

Table 4: Mean and standard deviation for the number of objectives solved for 25 runs of each fitness schedule. Means marked with asterisks $\left(^{*}\right)$ are not significantly different from each other, and are significantly different $(p<0.02)$ from those means without asterisks.

fixed and adaptive schedules. A two-tailed t-Test showed that the static and coevolving means are not significantly different from each other, and are significantly different $(p<0.02)$ from the fixed and adaptive means.

One of the motivations behind using coevolutionary search is the notion that the problem difficulty is ad-. justed automatically, rather than having to manually specify it. To get a sense of how coevolution accomplished this, Figure 20 shows four plots (one for each target objective), each containing 25 curves (fitted using a fourth-order polynomial). The plots show how the values of the best target vectors found in each generation fluctuated during the run. The thick curve represents the run that found a compliant circuit (i.e., it solved $(60.0,1.0,1.0,10.0\rangle)$.

What is most striking is the way coevolution, within the first few generations, reduced the demands for gain performance because it was the most difficult criterion to meet. Just as rapidly, the other three objectives were made more demanding because they were relatively easy to satisfy. Then as the circuit population scored better in gain, it did so at the expense of power and linearity: both power and linearity are seen peaking near generations $1000-2000$.

From the results it is seen that static and coevolutionary fitness schedules outperformed the fixed and adaptive schedules. Although is not completely clear why this happened, we can offer potential advantages of the static and coevolutionary schedules relative to the fixed and adaptive schedules. First, because a static fitness function induces a fitness landscape that never changes over the course of evolution there is never the possibility of getting "thrown off" a gradient (as would be the case if the fitness function changed). Second, we designed coevolution so that it would keep the level of problem difficulty near the leading edge of circuit proficiency. Developmental theory suggests (e.g., [1]) that keeping task difficulty in line with solution performance aids learning. Third, the fixed and adaptive schedules are potentially "handicapped" by the somewhat arbitrary choice of manually-crafted schedules. 


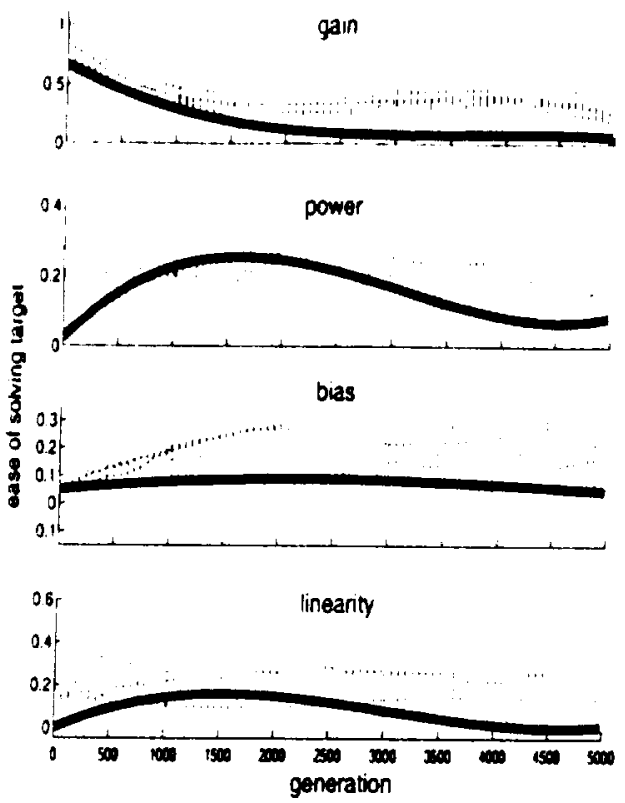

Figure 20: Highest fitness target vector values over the course of all coevolution runs. The $y$-axes represent the difficulty of the objective with 0.0 being the target (or most difficult) value, and 1.0 being the easiest objective value. The thick curves represent the run that found a compliant circuit. Curves were fitted using a fourthorder polynomial, and therefore sometimes appear above 1.0 and below 0.0 .

\section{Discussion}

As a step towards demanding space-related applications, we have presented encouraging results of an evolvable hardware system capable of automatically desinging analog circuits. We showed that a linear circuit representation and evolutionary search can automatically produce circuit designs of low to medium difficulty in two applications. Detailed simulations of the evolved designs suggest that all are electrically well behaved and thus suitable for physical implementation. The circuit representation method devised permits a wide range of circuits to be constructed, and results in a construction process that is unburdened with repair operations. In addition, the representation is syntactically closed, making it well suited for evolutionary search. For other applications, the instruction set can be easily extended to incorporate other devices not mentioned, such as CMOS transistors. The main limitation of our approach is the inherent restriction on circuit topologies. Such restrictions can be overcome by augmenting the instruction set, and this is one line of investigation we are pursuing. To gain performance on par with circuits designed by engineers, it will be necessary to place further constraints into the fitness functions. For example, practical amplifiers are typically judged by a dozen or so specifications. To evolve an amplifier that would perform as well would require using a multiobjective fitness function that accounts for each spereitication. Progeress towards this gond wiss matle in the results from coevolutionary search.

Dynamic: fituess schedule's can help evolutionary search because they encourage the population of circuits to follow potentially better trajectories through the solution space. Such trajectories could guide evolution in many ways, for example they comlel amplify weak gradients in the? fitness landscape, "steer around" meta-stable solution states [16], and usefully decompose or simplify the problem by providing partial reinforcernent for intermediate solutions [4]. As an illustration, an amplifier made up of a single wire has excellent performance in terms of bias, linearity and power dissipation, but has zero gain. Adding some components to the circuit might increase the gain, but only at the cost of a dip in performance on the other three criteria. Thus, if evolved with a static fitness schedule (assuming equally-weighted objectives), the single wire presents evolutionary search with a meta-stable state that is highly attractive and potentially quite difficult to escape. In contrast, the fixed fitness schedule in the present amplifier design task encourages all of the performance objectives (gain, power dissipation, bias, and linearity) to be solved in parallel by evolution. Likewise, coevolution tends to work on gain early in evolution and to scale back the requirements on bias, power and linearity until circuits are performing fairly well on gain.

In conclusion, static and coevolving fitness evaluations did relatively well in our amplifier design task. Based on our previous work in evolving amplifier designs, we suspected that the static technique would be able to solve this design task. We find it very encouraging that coevolution performed on par with static fitness schedules and intend to pursue coevolutionary search in future circuit design tasks, especially for electronic control applications.

\section{References}

[1] J.L. Elman, Incremental Learning, or the Importance of Starting Small, Tech. Rept. 9101, Center for Research in Language, University of California, San Diego, CA, 1991.

[2] G. Gielen, W. Sansen, Symbolic Analysis for Automated Design of Analog Integrated Circuits, Boston, MA: Kluwer, 1991.

[3] J.B. Grimbleby, "Automatic Analogue Network Synthesis using Genetic Algorithms," Proc. First Int. Conf. Genetic Algorithms in Engineering Systems: Innovations and Applications (GALESIA), 1995, pp. 53-58.

(4) G.L. Haith, S.P. Colombano, J.D. Lohn, D. Stassinopoulos, "Coevolution for Problem Simplifcation," Proc. 1999 Genetic and Evolutionary Com- 


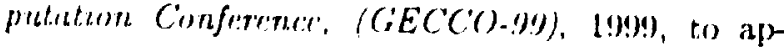
priatr.

[5] T. Hignuchi, M. Iwata, I. Kitjitani, H. Iba, Y. Hiraw, T. Furuya, B. Manderick, Evoluable Hardware and It.s Applications to Pattern Recognition and Fault Tolerunt Systems, (Lecture Notes in Computer Scienee), vol 1062, Berlin: Springer-Verlag, pp. 118135., 19966.

[6] T. Higuchi, M. Iwata, Eds., Evolvable Systems: From Biology to Hartware, Proc. of the First International Conference on Evolvable Systems, (Lecture Notes in Computer Science), vol 1259, Berlin: Springer-Verlag, 1997.

(7) J.H. Holland, Adaptation in Natural and Artificial Systems, Univ. of Michigan Press, Ann Arbor, 1975.

[8] D.H. Horrocks, Y.M.A. Khalifa, "Genetically Derived Filters using Preferred Value Components," Proc. IEE Colloq. on Linear Analogue Circuits and Systems, Oxford, UK, 1994.

[9] L.P. Huelsman, Active and Passive Analog Filter Design, New York: McGraw-Hill, 1993.

(10] J.R. Koza, F.H. Bennett, D. Andre, M.A. Keane, F. Dunlap, "Automated Synthesis of Analog Electrical Circuits by Means of Genetic Programming," IEEE Trans. on Evolutionary Computation, vol. 1, no. 2, July, 1997, pp. 109-128.

[11] J.R. Koza, F.H. Bennett, J.D. Lohn, F. Dunlap, M.A. Keane, D. Andre, "Use of ArchitectureAltering Operations to Dynamically Adapt a ThreeWay Analog Source Identification Circuit to Accommodate a New Source," in Genetic Programming 1997 Conference, J.R. Koza, K.Deb, M.Dorigo, D.B. Fogel, M. Garzon. H. Iba, and R.L. Riolo, (eds), Morgan Kaufmann, 1997, pp. 213-221.

[12] M.W. Kruiskamp, Analog Design Automation using Genetic Algorithms and Polytopes, Ph.D. Thesis, Dept. of Elect. Engr., Eindhoven University of Technology, Eindhoven, The Netherlands, 1996.

[13] J.D. Lohn, S.P. Colombano, "Automated Analog Circuit Synthesis using a Linear Representation," Proc. of the Second Int'l Conf on Evolvable Systems: From Biology to Hardware, Springer-Verlag, Berlin, 1998, pp. 125-133.

[14] J.D. Lohn, S.P. Colombano, "A Circuit Representation Technique for Automated Circuit Design," IEEE Trans. on Evolutionary Computation, to appear.

(15) E.S. Ochotta, R.A. Rutenbar, L.R. Carley, "Synthesis of High-Performance Analog Circuits in ASTRX/OBLX," IEEE Trans. Computer-Aided Design, vol. 15, pp. 273-294, 1996. [li] C.D. Rosin, R.K. Belew, Ne'w Methods for Competetive: Coevolution. Texh. Rept. CS9ti-491, Department of Computer Science? and Engineering, University of California, Sin Diego, 1996.

117) E. Sinchez and M. Tomilssini, Eds., Toward Evolvable Hardware: The Evolutionary Engineering Approach, (Lecture Notess in Computer Science), vol 1062, Berlin: Springer-Verlag, 1996.

[18] M. Sipper, D. Mange, A. Perez-Uribe, Eds., Evolvable Systems: From Biology to Hardware, Proc. of the Second International Conference on Evolvable Systems, (Lecture Notes in Computer Science), vol 1478, Berlin: Springer-Verlag, 1998.

[19] M. Sipper, D. Mange, Eds., Special Issue on Evolvable Hardware IEEE Transactions on Evolutionary Computation, vol 3, no 3, 1999.

[20] G.J. Sussman, R.M. Stallman, "Heuristic Techniques in Computer-Aided Circuit Analysis," IEEE Trans. Circuits and Systems, vol. 22, 1975.

[21] A. Thompson, "An Evolved Circuit, Intrinsic in Silicon, Entwined with Physics," in T. Higuchi, M. Iwata, Eds., Evolvable Systems: From Biology to Hardware, Proc. of the First International Conference on Evolvable Systems, (Lecture Notes in Computer Science), vol 1259, Berlin: Springer-Verlag, pp. 390-405, 1997.

[22] J. Villasenor, W. Mangione-Smith, "Configurable Computing," Scientific American, vol 276, no 6, pp. 67-71, June 1997.

[23] R.S. Zebulum, M.A. Pacheco, M. Vellasco, "Comparison of Different Evolutionary Methodologies Applied to Electronic Filter Design," 1998 IEEE Int. Conf. on Evolutionary Computation, Piscataway, NJ: IEEE Press, 1998, pp. 434-439. 